\title{
Régimes du visuel et transformations de l'allégorie
}

\author{
Christian Vandendorpe \\ Université d'Ottawa
}

Protée , 33.1, printemps 2005, p. 25-38.

Il y eut des époques et des sociétés où les images étaient dotées d'une efficacité magique : c'était l'âge d'or de l'idole ou premier âge de l'image - qui sera suivi par l'âge de l'art puis du visuel, selon la périodisation proposée par Régis Debray (1992). La vénération de l'idole faisait participer le croyant de la force spirituelle du dieu qu'elle représentait. En dehors de ce régime magique, devenu rare aujourd'hui, rien ne garantit au créateur d'une image que celle-ci sera examinée dans toutes ses dimensions symboliques et esthétiques. Chacun a probablement fait l'expérience d'être passé devant une image des centaines de fois sans la voir au sens fort du terme, sans se questionner sur ce qu'elle signifie, sur le projet de communication ou d'expression qui l'avait fait naître. Cela vient du fait que l'image ne fait pas signe de la même façon que le langage. Un mot ou un graffiti sur un mur suscitent un réflexe de lecture chez une personne alphabétisée, car nos mécanismes de traitement cognitif sont automatiquement sollicités par le langage, qui est toujours censé véhiculer un message et donc mériter un traitement, voire susciter une réponse. Le langage est un médium actif, qui sollicite directement le lecteur ou l'auditeur; l'image attend du passant ou du spectateur qu'il s'intéresse à elle.

Le créateur visuel doit donc déployer des stratégies complexes s'il veut que son image soit véritablement vue, avec tout le recueillement et toute l'intensité dont le regard est capable. Pour cela, il importe au départ que l'image ne se 
confonde pas avec le monde naturel et qu'elle ne soit pas non plus un simple élément d'un décor sur lequel l'œil peut glisser superficiellement sans même prendre conscience de son existence. Pour retenir l'attention du spectateur, l'image doit d'abord interpeller par l'effet qu'elle produit et les émotions qu'elle est à même de susciter. Et cela dépend du jeu des couleurs, des lignes et surtout du sujet représenté. Même pour un peintre aussi cérébral que Magritte, l'effet est l'enjeu fondamental de la peinture, une image n'ayant du sens que si « elle agit sur le récepteur, bouleversant ses habitudes mentales, provoquant un questionnement, modifiant sa façon de voir le monde » (Everaert-Desmedt, 1990 : 90).

Dans un deuxième temps, il faut que l'image soit perçue comme porteuse de signification, qu'elle enclenche chez le spectateur une activité sémiotique. C'est seulement lorsqu'il y a cet "arrêt sur image » que peut prendre place un traitement cognitif en profondeur, susceptible d'entraîner une traduction élaborée $d u$ percept visuel sous la forme de pensées, du moins dans le mode classique du rapport à l'image. Une fois exprimées par des propositions verbales, ces perceptions visuelles permettront éventuellement à d'autres personnes de participer de la même expérience de construction de sens. En se multipliant, elles en arriveront à conférer à l'objet une sorte d'aura, qui fonctionnera à la façon d'une matrice de réception esthétique. C'est ainsi que certaines images laissent des traces durables dans la mémoire individuelle et collective. Dans le meilleur des cas, elles finiront par accéder au statut d'«icônes culturelles » et seront inlassablement citées, commentées, reproduites ou pastichées. Il ne s'agit toutefois pas d'une infinie répétition du même, car, comme le note Rudolf Wittkower, «chaque génération produit sa propre interprétation des anciens symboles par lesquels elle est attirée et avec lesquels elle se sent des affinités, mais aussi crée de nouveaux symboles en utilisant, modifiant et transformant ceux du passé » (p. 184).

Tout comme il n'y a pas d'image "naturelle », il n'existe pas de perception brute ou naïve des images, qui projetterait dans notre cerveau une réplique fidèle de ce qui est vu. Une image est toujours perçue à travers un jeu complexe de codes esthétiques et de références culturelles. De même, elle implique, de la part du créateur, la mise en œuvre de codes et de contenus symboliques susceptibles d'être reconnus et appréciés par les membres de la société à laquelle il s'adresse.

\section{Trois grands régimes cognitifs}

On ne lit pas un poème comme un roman, et un roman sentimental n'appelle pas le même type de lecture qu'un roman policier. Ce sont là des genres différents, dont les règles doivent être apprises. Les conventions génériques, en plus d'aider le créateur, aident le lecteur à instancier une attitude de réception aussi bien adaptée à l'oeuvre que possible. 
Même si les genres en peinture sont nettement moins marqués qu'en littérature, nous savons d'expérience que toutes les images ne suscitent pas la même activité de lecture et d'interprétation. Diverses caractéristiques sollicitent différemment l'intérêt du spectateur, le guident dans son exploration de l'image et l'aident à en garder une trace en mémoire. A titre heuristique, je propose de distinguer dans la lecture de l'image trois régimes cognitifs principaux : le narratif, le descriptif et le symbolique.

Sous le régime narratif, les données visuelles sont organisées afin de susciter chez le spectateur une démarche similaire à celle de la lecture d'un récit. Il s'agira donc pour le créateur de reproduire dans un tableau ou une sculpture une séquence d'actions ou d'événements. Celle-ci peut prendre la forme canonique du récit et représenter le début, le milieu et la fin d'une action. Mais le plus souvent, le tableau ou la sculpture ne feront qu'illustrer le moment caractéristique du récit, soit l'apex, à partir duquel le spectateur devra reconstruire mentalement les tenants et aboutissants. Le régime narratif convient particulièrement à des sociétés où circulent des récits connus de tous : épisodes de la bible, récits mythologiques, vies de saints. Devant ce genre de tableaux, le spectateur n'a qu'à puiser dans sa connaissance de l'histoire représentée pour pouvoir se livrer à une lecture active. Une fois le sujet identifié, il pourra retrouver le moment de l'histoire que le peintre ou le sculpteur a choisi de représenter, examiner attentivement les acteurs en présence, et évaluer l'adéquation du tableau au récit, aux divers plans de la posture corporelle, de l'expression du visage, des vêtements, du décor, etc. Les attributs des personnages ne sont pas choisis au hasard, mais servent à les identifier aussi sûrement qu'une désignation verbale. Comme le note lumineusement Michel Butor :

Bien des objets dans les tableaux anciens étaient des mots. Chaque saint était caractérisé par un ou plusieurs objets: Agnès par un agneau, Roch par un chien, Pierre par une clef, Jérôme par un livre et un lion. Pour les martyrs, c'est très souvent l'instrument de leur supplice, ou bien la partie suppliciée. (1951:51)

Sous ce régime, l'image est un objet à lire, à interpréter et à commenter. Elle peut aussi facilement être reconstruite en mémoire et évoquée dans ses détails. Une miniature des frères Limbourg servira d'exemple : Le Jardin d'Eden, extraite des Très riches heures du duc de Berry ${ }^{1}$ (Figure 1). Réalisée vers 1410, l'image montre «successivement» la tentation d'Ève par le serpent, puis celle d'Adam par Ève, suivie de la sévère remontrance que Dieu leur adresse et leur expulsion subséquente du paradis. Les quatre séquences clés du récit biblique sont insérées

\footnotetext{
${ }^{1}$ Folio 25v. http://www.christusrex.org/www2/berry/f25v.html
} 
dans une même composition, à l'intérieur d'une bulle dont la forme circulaire convient bien à l'évocation d'un temps et d'un espace mythiques. Grâce à la connaissance préalable qu'il a de ce récit, le spectateur peut facilement repérer les divers épisodes et les replacer dans leur ordre chronologique, en effectuant une lecture de gauche à droite. Le tableau se lit donc avec la même rigueur qu'une phrase, en imposant à la lecture un ordre syntaxique axé sur la temporalité.

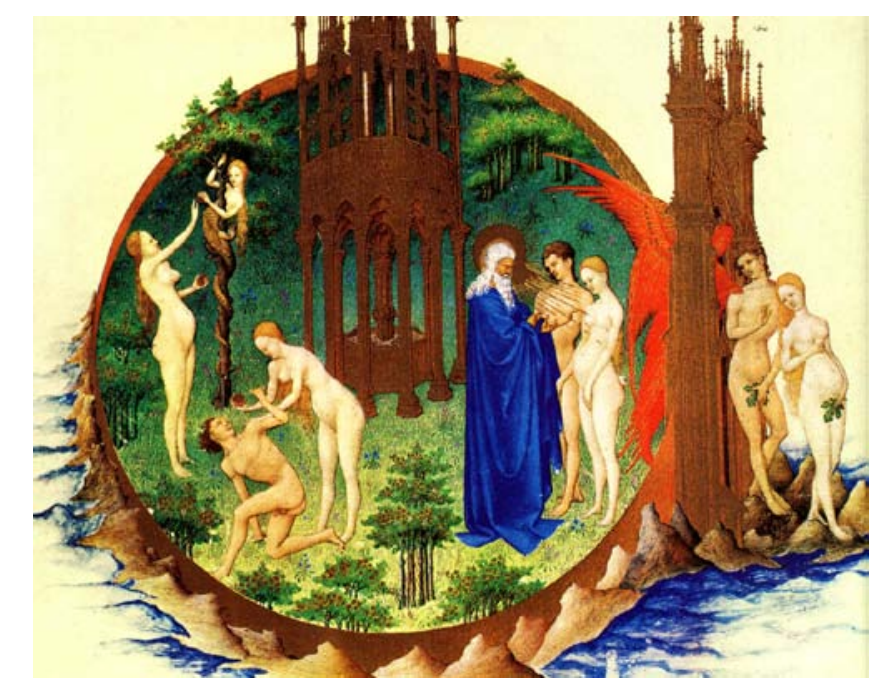

Figure 1. Le Jardin d'Eden. Miniature des frères Limbourg pour Les très riches heures du duc de Berry (1410)

Ce mode d'organisation est aussi intéressant pour le créateur que pour le spectateur du tableau. En effet, arrivé au terme de la reconstitution de la séquence narrative, ce dernier se sentira gratifié d'avoir réussi à produire du sens à partir d'une image apparemment muette. Et cette réussite le confortera dans sa qualité de membre à part entière d'une communauté interprétative dont il maîtrise assurément les codes. Quant au peintre, le régime narratif lui garantit que le spectateur, dans son activité sémiotique de repérage de la séquence des actions, examinera son image avec une attention redoublée, sans en négliger aucune section ni aucun indice. Ce régime, en s'appuyant sur des clés de lecture largement présentes dans une société - particulièrement en ce qui a trait aux récits bibliques et aux vies de saints -, maximise ainsi la communication esthétique entre l'artiste et son public.

Le régime descriptif se situe à un pôle opposé. Il ne s'agit plus ici de raconter une histoire, mais de jouer sur la représentation en imposant une image à l'imaginaire du spectateur grâce à sa force d'évocation, son réalisme ou sa beauté. 
L'opposition entre le descriptif et le narratif a été mise en évidence par Gotthold Ephraïm Lessing, dans son essai Laocoön, publié en 1766. Prenant à contre-pied le précepte de l'antiquité classique voulant que la poésie imite la peinture (Horace : "Ut pictura poesis »), Lessing suggère que ces deux formes artistiques ne doivent pas s'imiter mutuellement, mais bien plutôt miser sur leurs spécificités, qui sont radicalement différentes. Le poème, qui se développe dans le temps, excelle à représenter des actions, alors que le tableau ou la sculpture, qui relèvent de la dimension spatiale, conviennent surtout à la description des corps: ce n'est qu'accessoirement que la poésie peut aussi suggérer des corps, et la peinture évoquer des actions. Toute tentative de subordonner la poésie à la description picturale ne peut donc que l'enliser dans l'insignifiance; de même, en se voulant trop narrative, la peinture risque fort de sombrer dans l'artifice. Abondamment cité et discuté, l'essai de Lessing inaugurera la réflexion moderne sur l'esthétique et contribuera à l'autonomisation de la peinture.

Les tableaux axés sur le descriptif existaient bien avant Lessing et ils ont peut-être même été une des grandes conquêtes de la peinture, après les idoles, si on ajoute foi à l'anecdote voulant que des oiseaux auraient tenté de picorer une grappe de raisins peinte par Zeuxis (-400).

La description en peinture ou en sculpture offre une prise naturelle au regard du spectateur, qui examinera l'adéquation au réel, la précision du dessin et des couleurs, la richesse du détail. Un portrait retiendra cependant d'autant plus

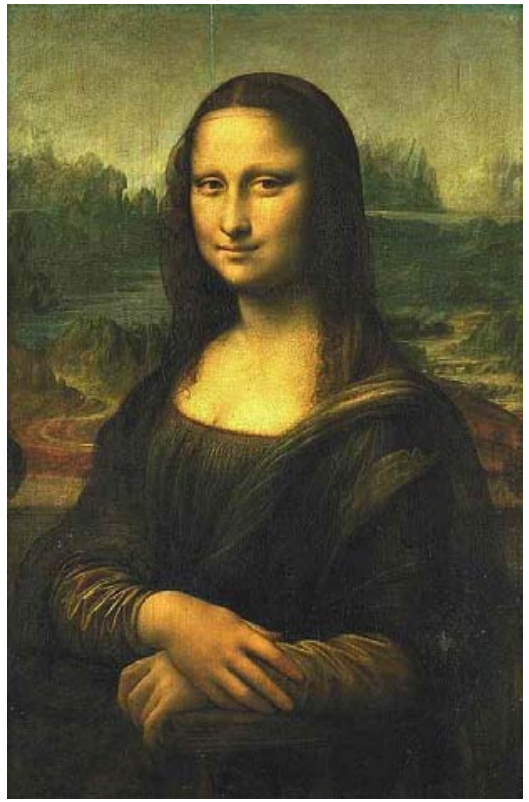
l'attention qu'il présente un personnage hors du commun dans une pose mystérieuse et sur un arrière-fond intéressant. La combinaison de ces caractéristiques a contribué à faire de « La Joconde » un des sommets de la veine descriptive, qui a fasciné des générations de spectateurs et de critiques (Figure 2). Le critique d'art E.H. Gombrich attribue le mystérieux effet que produit ce tableau à divers artifices utilisés par Léonard de Vinci (1995 : 303). Le premier est l'emploi savant de ce qu'il appelle le «sfumato » - ou plutôt ici le «fondu » - autour des lèvres et des yeux, qui plonge dans une ombre imprécise les zones du visage qui auraient pu aider à identifier l'humeur de Mona Lisa et la façon dont elle nous regarde. Un autre artifice, bien plus audacieux, est d'avoir délibérément fracturé l'arrière-plan, en dessinant l'horizon sur le côté gauche beaucoup plus bas qu'à droite. Il s'ensuit que lorsqu'on regarde la femme du côté gauche, elle semble plus grande que si l'on se fixe sur le côté droit 
du tableau, et cette apparence de dissymétrie affecte aussi son visage. Ces divers effets fascineront d'autant plus le spectateur de ce tableau qu'il en fera une exploration visuelle plus minutieuse : loin de s'abolir dans la tautologie, écueil de la peinture réaliste, le mode descriptif débouche ici sur un cercle herméneutique soigneusement élaboré.

Les régimes descriptif et narratif ne doivent évidemment pas être vus comme des compartiments étanches, mais plutôt comme des traits génériques qui peuvent se combiner dans des proportions variables. Il en va de même du régime symbolique. Ce dernier désigne ici un vaste ensemble de productions pour lesquelles le créateur a principalement recours à des symboles afin d'intriguer le spectateur et de l'amener à rechercher activement la signification cachée d'éléments représentés dans le tableau. Cette façon de stimuler le parcours interprétatif du spectateur contribue, elle aussi, à produire des événements de sens et à rendre un tableau mémorable.

\section{Expressions du symbolique}

La notion de symbole est complexe et a suscité de nombreux travaux (Todorov, 1997; Eco, 1988). Loin d'être univoque, la signification de ce terme varie selon les époques et les contextes disciplinaires où il est utilisé. Sa signification actuelle s'est fixée au XIXe siècle, même si le mode symbolique était pratiqué par les artistes depuis toujours. Le symbole est une image d'une réalité concrète ou une qualité du monde physique (telle la couleur), qui, en plus de signifier son référent, est susceptible de suggérer un faisceau de significations abstraites qui en dérivent. Cette dérivation se fait nécessairement de manière indirecte, à la suite d'une opération cognitive de type rhétorique : métaphore, métonymie, déplacement, condensation, association, etc. Le mode symbolique recouvre des opérations fondamentales de l'esprit humain, telles qu'elles sont observables notamment dans le rêve, où elles atteignent à leur état de fluidité maximale (Freud, 1900).

Le tournant épistémique inauguré par la révolution romantique se cristallise dans l'opposition qu'elle instaure entre symbole et allégorie. À la suite de Goethe, le symbole est alors paré de toutes les qualités, tandis que l'allégorie est accablée de tous les défauts. Ce discrédit jeté sur l'allégorie se maintiendra durablement, à tel point que cette figure répugne toujours à la pensée contemporaine : « les allégories sont au domaine de la pensée ce que les ruines sont au domaine des choses » (Walter Benjamin, 1925 : 191). Le symbole et la fonction symbolique, en revanche, sont à la base de la révolution freudienne, qui aurait été impossible sans que soit communément admise la légitimité de recourir à des façons obliques de signifier. Anticipant sur les découvertes de la pragmatique, cette notion de symbole a permis « de faire entrer dans l'ordre des signes interprétables des don- 
nées qui, jusque-là, étaient d'ordre privé et ne pouvaient être exprimées ou qui étaient rejetées comme relevant d'une mentalité magique incompatible avec le triomphe de la rationalité » (Vandendorpe, 1999 : 83).

En même temps, l'opposition romantique entre symbole et allégorie est quelque peu forcée, car les deux termes ne relèvent pas du même plan

d'expression. En matière de langage, le symbole est tout entier contenu dans un seul terme (par exemple : lion, faucille, rouge) alors que l'allégorie exige au moins une proposition pour se développer («L'Allégorie habite un palais diaphane», selon l'expression allégorique de Lemierre, citée dans le Grand Larousse). Elle peut même se poursuivre à travers une fable (par exemple chez Florian, IV, 20, « Le voyage »), voire un roman tout entier (Le roman de la rose). Cela explique que l'allégorie soit considérée depuis Quintilien comme une figure de rhétorique, à la différence du symbole, qui n'a été considéré tel qu'à une époque récente. Une allégorie est une création syntagmatique, alors qu'un symbole est une entité virtuellement ouverte qui exige un contexte pour se mettre à fonctionner.

Il ne fait pas de doute que, en littérature, la faveur de l'allégorie a beaucoup varié au cours des âges. Nous examinerons s'il en est de même dans les arts visuels.

\section{L'allégorie classique}

L'Allégorie à la Vierge, de Hans Memling (1480), est un petit tableau de $38 \mathrm{x}$ $32 \mathrm{~cm}$, exposé au musée parisien Jacquemart-André (Figure 3). Le spectateur qui en approche est d'abord intrigué par la posture de la jeune femme et le paysage qui l'entoure, qui n'ont pas d'équivalent dans la nature. Cette image ne peut donc pas être vue comme un récit ni comme une description, mais exige que le spectateur réussisse à opérer une traduction des signes présentés, afin d'en dégager la signification symbolique.

Un examen minutieux pourra fournir une clé de lecture. Il s'agit pour cela de combiner divers indices convergents : le regard chastement dirigé vers le bas et les mains croisées devant soi dénotent chez la femme une attitude extrêmement réservée. L'améthyste géante qui enserre le bas du corps est, selon le Dictionnaire des symboles, « une pierre de tempérance qui garde de toute ivresse »; cette pierre, qui est portée par les évêques, serait aussi «le symbole de l'humilité, parce qu'elle est de la couleur de la violette ». Cette couleur étant aussi celle du vêtement, il est clair que ces connotations sont à rapporter à la femme, par métonymie. Au bas du rocher, deux lions redoutables semblent monter la garde. Très fréquent dans l'iconographie chrétienne, cet animal est un symbole de force et de 


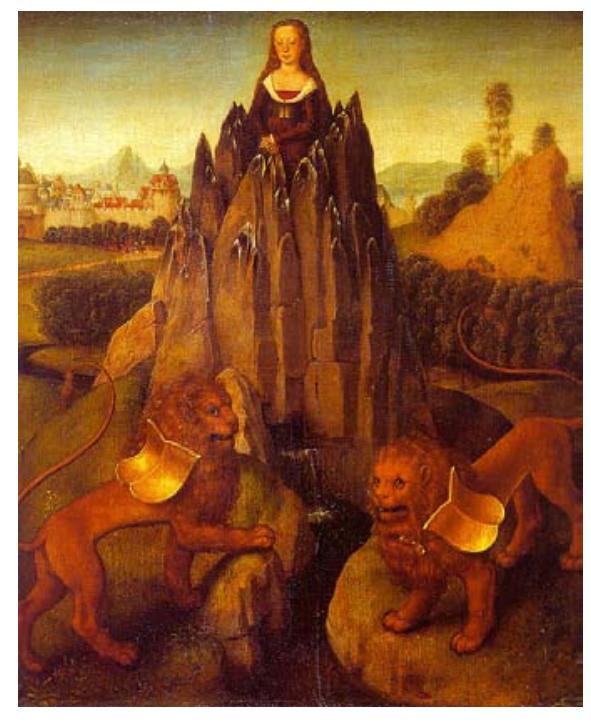

courage au service du roi : il est donc clair que leur présence ne constitue pas une menace mais une protection pour la jeune femme. Pour preuve, ils portent sur le dos un écu indiquant leur qualité de chevaliers servants. Enfin, on aperçoit une source d'eau pure jaillissant du rocher, symbole de vie éternelle.

L'effet de ces divers symboles est de créer une isotopie de la pureté et de la virginité, que protègent d'infranchissables remparts. Ces caractéristiques une fois attribuées au personnage dominant qu'est la dame, le spectateur peut dès lors effectuer le mouvement de traduction exigé par le tableau et voir dans celle-ci une allégorie de la virginité. Tout comme dans la lecture d'une allégorie verbale, on est ainsi passé d'une scène imagée fort concrète (lions, rocher, couleur violette, paupières baissées) à l'idée abstraite que celle-ci vise à exprimer. Ici encore, le parcours interprétatif débouche sur une signification unifiée qui facilitera la mémorisation et le commentaire.

Il faut toutefois noter que cette interprétation n'est pas donnée d'emblée et ne sera évidente que si l'on est déjà familier avec les symboles utilisés. Pour beaucoup de spectateurs d'aujourd'hui, cette image vue sans son titre actuel ni aucun commentaire pourrait bien rester une énigme, ainsi que l'affirme la Web Gallery of Art $^{2}$.

C'est pour assurer la lisibilité des allégories que l'époque classique fera le plus grand cas de l'Iconologia de Cesare Ripa (1560-1623). Qualifié par Michel Pastoureau de «bric-à-brac symbolique » (522), cet ouvrage connaîtra de nombreuses rééditions, illustrées par divers graveurs et fera sentir son influence jusqu'au XIX ${ }^{\mathrm{e}}$ siècle. La première édition (1593), traduite en français par Jean Baudoin (1644), propose dans sa Préface une véritable propédeutique de l'allégorie 3.

Selon l'auteur, la peinture peut avoir recours à deux sortes d'images. La première est constituée par les figures mythologiques « qui ne sont à proprement parler que des voiles ou des vêtements propres à couvrir cette partie de la Philo-

2 http://www.kfki.hu/ arthp/html/m/memling/2middle3/15allego.html

${ }^{3}$ Pour des raisons techniques, nous avons repris ici l'illustration de la Vérité tirée de l'édition italienne plutôt que de la française. 
sophie, qui regarde ou la génération des choses naturelles, ou leur corruption, ou la disposition des Cieux, ou l'influence des Astres, ou la solidité de la terre » (Préface). Une figure mythologique est donc d'emblée allégorique, car elle exprime autre chose qu'elle-même : Saturne est ainsi l'image du temps ; Vénus est celle de «l'union de la première matière avec la forme, d'où lui vient la perfection ».

Une autre classe d'images est celle des « choses qui sont en l'homme même », soit les pensées et «tout ce qui peut être signifié par les paroles ». Ces images doivent être organisées autour d'un personnage, en vertu du principe aristotélicien voulant que «l'homme est la mesure de toutes choses ». Enfin, cette figure doit être accompagnée de divers attributs susceptibles d'orienter l'esprit du spectateur dans la direction du sens suggéré.

Ripa rejette les symboles communs et trop faciles à déchiffrer, comme de recourir à un lion pour représenter la vaillance et le courage. Il préfère des figures composites et travaillées, inspirées de l'art romain des médailles. La personnification recourt dans la plupart des cas à la figure féminine, l'homme n'étant évoqué que pour représenter la force, le courage, l'honneur, le péché, la tromperie... Ce clivage n'est pas innocent. L'image, en effet, doit d'abord séduire par le contenu représenté afin de mieux mettre à distance la froideur inhérente aux objets.

Parmi les quelque 430 allégories imagées et commentées que cet ouvrage propose, on trouve notamment la figure incontournable de la Vérité (Figure 4).

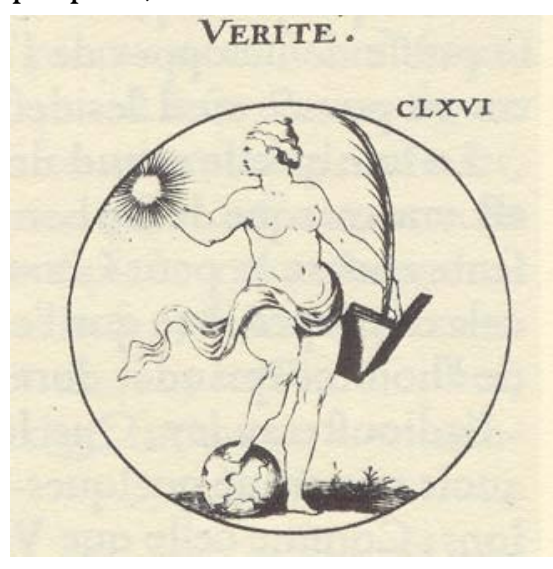
Celle-ci est représentée sous la forme d'une femme nue, afin de montrer, selon les auteurs, que «la naïveté lui est naturelle et qu'elle n'a pas besoin d'explication pour se faire entendre » (195); elle «tient et regarde un Soleil, qui est Dieu, source de toute lumière, et la même vérité »; le livre "qu'elle tient ouvert signifie que, dans les écrits des bons auteurs qui nous apprennent les sciences, se trouve la vérité des choses »; «la branche de palme, que la vérité n'a pas moins de force à se raidir contre les efforts qui s'y opposent, qu'en a la palme à se relever, plus on essaie de l'abattre »; enfin, la Vérité « est plus forte que toutes les choses du monde : aussi est-ce pour la même raison qu'elle foule un Globe de l'un de ses pieds » (196).

Chaque figure comporte plusieurs attributs, et c'est de leur combinaison que provient la signification. Celle-ci toutefois - et c'est le propre des images n'est jamais autonome au point de pouvoir être déchiffrée avec assurance. Aussi la figure est-elle toujours accompagnée de son inscription : 
Ces images, si la disposition en est bonne, et la manière ingénieuse, ont je ne sais quoi de si agréable qu'elles arrêtent la vue et font aussitôt désirer à l'esprit de savoir ce qu'elles signifient. Mais surtout cette curiosité se redouble par leur inscription. En effet, il faut nécessairement qu'elles en aient une, si ce n'est quand elles sont en forme d'énigme, pour ce que, sans la connaissance du nom, il est impossible de parvenir à la chose signifiée. (Préface)

Pour Baudoin et Ripa, le plaisir que ces figures sont censées procurer provient de l'adéquation que l'on pourra découvrir entre les attributs imagés et le concept abstrait désigné par l'inscription. En somme, la figure ainsi conçue énonce une question (« pourquoi est-elle une allégorie de la Vérité?) dont on ne découvrira la réponse qu'à la suite d'un parcours de type propositionnel appliqué aux divers éléments de l'image. Le principe à l'œuvre est le même que dans la fable, où la morale résume et reformule à un niveau général et abstrait la leçon découlant du récit. Au plan du travail cognitif, il y a donc va-et-vient entre le niveau conceptuel dénoté par l'inscription et le contenu descriptif de l'image. L'image concrète doit permettre à l'esprit de remonter jusqu'au concept et cela se fait en puisant dans un vaste répertoire de symboles codés. Tout le savoir culturel de l'époque est susceptible d'être convoqué à cette fin : le chat pour la Liberté, le serpent pour la Perfidie, la robe de pourpre pour l'Honneur, etc. Dans de nombreux cas, l'image renvoie aussi à des dictons, locutions figées, clichés et stéréotypes. Ainsi, la vérité est-elle souvent qualifiée de «nue », « lumineuse » et « aveuglante ». L'image de la Reconnaissance est illustrée par un éléphant parce que « cet animal n'oublie jamais le bien qu'il a reçu » (86) - ce qui montre que l'expression " avoir une mémoire d'éléphant » était déjà en usage à l'époque. L'allégorie de l'Art a une robe de couleur verte «à cause de l'espérance qu'ils [les artistes] ont, ou d'en tirer de la gloire, ou d'en avoir du profit » (21). Parfois une image est tout entière construite sur un seul dicton, voire un stéréotype discutable, comme l'allégorie intitulée "Peine perdue », qui représente un homme à la peau foncée tenant une serviette. L'explication qu'en donne Ripa est exemplaire du fonctionnement allégorique classique: «Cette figure n'a pas besoin d'être expliquée, puisque la chose qu'elle démontre est si véritable qu'elle a donné lieu au Proverbe qui dit 'Qu'à laver le corps d'un More, pour le faire devenir blanc, on n'y perd que la lessive' » (167). L'allégorie visuelle affirme ici clairement sa nature propositionnelle et son inféodation au langage. 


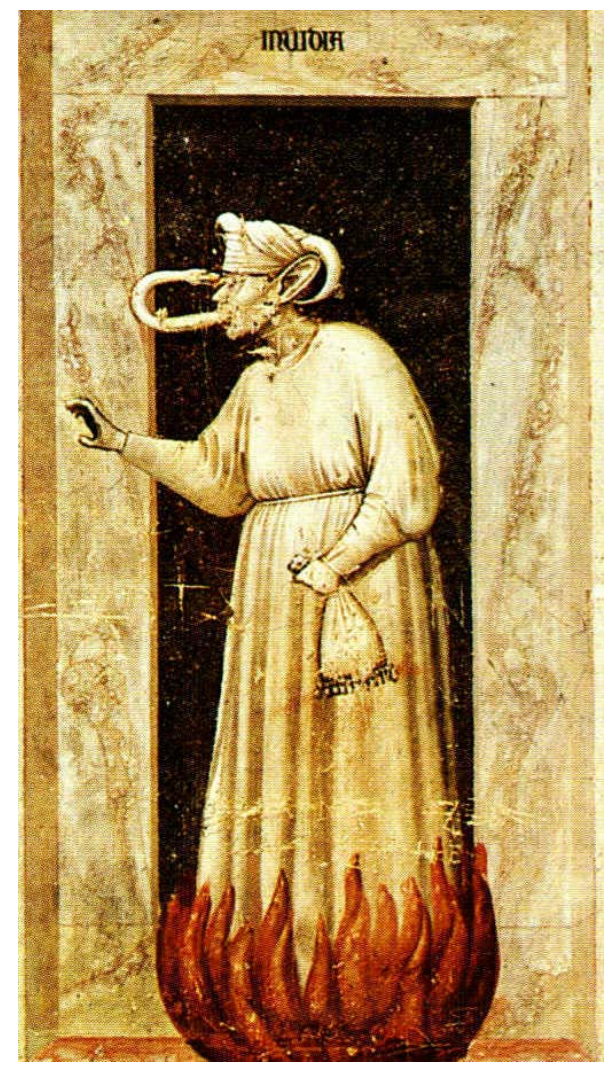

Ce fonctionnement de l'allégorie était déjà fixé à l'époque de Giotto, dans les allégories des vices et des vertus qu'il avait peintes en 1305 pour la chapelle des Scrovegni à Padoue. L'Envie (Figure 5) y est représentée par une vieille femme dont la langue démesurément allongée se replie sur elle-même comme un serpent. Son oreille est également démesurée, indiquant l'excès d'attention qu'elle prête aux commérages. Enfin, la vieille se tient debout sur un feu ardent. Tous ces traits aboutissent à une image artificielle et composite, sans référent dans la nature, au même titre que la dame de Memling. L'allégorie ne se comprend que si, après avoir trouvé la clé de lecture dans l'inscription située au-dessus du tableau, on déchiffre ensuite les divers symboles en les rapportant à des expressions métaphoriques empruntées au langage usuel : on retrouvera ainsi les expressions «brûler

d'envie » et « avoir une langue de serpent ».

Ce jeu de transcodage linguistique est poussé encore plus loin dans le tableau des «Proverbes flamands » de Pieter Brueghel ${ }^{4}$. Confronté à cette toile, le spectateur contemporain pourra, selon les cas, ne pas trouver un seul proverbe ou en identifier plusieurs dizaines. Tout dépend de la connaissance qu'il possède du discours social en vigueur à l'époque et de sa capacité à déchiffrer des énigmes.

En obligeant ainsi le spectateur à retrouver les lieux communs que l'image illustre, l'allégorie classique affiche clairement sa subordination au langage qui, seul, peut produire un effet de vérité.

\section{Ruine de l'allégorie}

Cette conception de l'allégorie sera prise à partie par l'ouvrage de Lessing évoqué plus haut. Avec la nouvelle sensibilité qui déferlera bientôt sur les pays européens à l'aube du XIX siècle, les peintres se tourneront vers d'autres formes d'expression symbolique. Au lieu d'un bric-à-brac d'attributs codifiés, le peintre

\footnotetext{
${ }^{4}$ http://www.inter-coproprietes.com/culture/Galerie/bruegl01.jpg
} 
romantique cherchera alors à renouer avec le mouvement, le naturel, le sentiment. Un bel exemple de ce renouveau de l'allégorie est donné par le tableau d'Eugène Delacroix, La Liberté guidant le peuple (1830). D'abord intitulé Une barricade, le tableau a pour sujet l'événement historique que fut la révolution de 1830 à Paris, qui chassa le roi Charles X, exécré pour son autoritarisme. Le drapeau est le seul symbole apparent. Mais, comme le note Nadeije Laneyrie-Dagen, «les personnages sont choisis pour leur portée symbolique» (2002: 31). De fait, on trouve parmi eux les prototypes du bourgeois, de l'ouvrier, du gamin de Paris, tous représentés d'une façon vivante et fort éloignée des personnages mythologiques ou costumés à la romaine auxquels avaient recours les peintres antérieurs ${ }^{5}$. Seule la femme «Liberté » portant le drapeau rappelle les personnages allégoriques, avec sa taille plus grande que nature et les seins découverts. Mais son regard et son attitude, saisies en plein mouvement, n'ont rien des personnages figés de l'allégorie classique. Une lecture symbolique est certes encore possible, en ce sens que le tableau prétend illustrer des réalités abstraites (Liberté, peuple) : une telle lecture est déjà tout entière contenue dans le titre. Le tableau possède cependant une existence en soi, car il constitue un authentique fragment narratif et descriptif, ancré dans une réalité historique et géographique, avec les tours de Notre-Dame de Paris visibles dans le lointain. L'allégorie est descendue du ciel éthéré de l'abstraction pour s'incarner dans des personnages de chair et de sang.

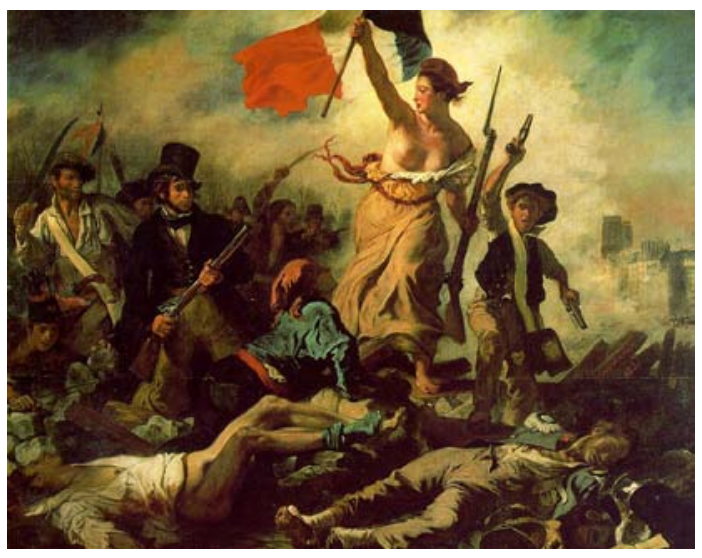

$\mathrm{Au} \mathrm{XX}^{\mathrm{e}}$ siècle, l'image franchira un pas supplémentaire dans son émancipation du langage. Après Picabia, qui jouait sur les rapports de dissonance entre le titre du tableau et le sujet représenté, Magritte se livrera à une exploration systématique des rapports entre «les mots et les images ${ }^{6}{ }^{6}$, rapports qu'il tentera de faire exploser. Le plus souvent, les mots sont mis en contradiction radicale avec l'objet représenté, comme dans le tableau «La trahison des images », où le dessin d'une pipe est accompagné de la légende Ceci n'est pas une pipe, qui a inspiré tant

${ }^{5}$ On comparera par exemple avec le tableau de Rubens, Allégorie sur les bénédictions de la paix, 1629-30. http://www.artchive.com/artchive/R/rubens/peace.jpg.html

6 Titre d'un article de René Magritte publié dans La Révolution surréaliste, Paris, $\mathrm{n}^{\circ}$ 12, 15 décembre 1929. 
de commentaires qu'il est inutile d'y revenir ici (voir notamment Foucault 1973). Mais, chez cet artiste, les rapports entre les mots et les images peuvent se faire plus retors, voire franchement pervers. Ainsi, dans Le miroir magique, les mots " corps humain » sont inscrits sur un miroir, comme si la réalité que devait refléter sa surface était soudainement transmuée en du langage. Les titres sont étudiés pour semer la confusion plutôt que pour éclairer la lecture de l'œuvre : « Loin de nous donner la clef du tableau, le titre relance le processus interprétatif, il empêche la fixation de la croyance, il maintient le doute, le mystère.» (EveraertDesmedt, 1994 : 130-131). En jouant ainsi sur les titres et les impossibilités logiques, toute cette œuvre vise à la déconstruction des liens unissant jusque-là représentation visuelle et langage. Soumis à ces heurts violents, qui nient le sens commun et réfutent sa compréhension du monde, le spectateur ne peut que tenter de se raccrocher à des explications plus ou moins satisfaisantes.

Au-delà du langage, c'est même le lien de l'image avec le sens qui est visé par cette oeuvre. Rejetant explicitement toute interprétation qui chercherait un sens caché dans ses tableaux, Magritte prétend vouloir :

[...] peindre des images qui montrent des choses du monde dit réel de manière à ce qu'elles ne correspondent plus à des idées ou à des sentiments. J'évite, par exemple, de peindre une figure qui représenterait l'idée de la Justice, avec toutes les interrogations liées à une telle idée. (1994 : 133)

Le peintre, selon Magritte, ne devrait rien donner à penser par ses tableaux. Jamais le mode symbolique n'avait été aussi radicalement pris à partie.

En même temps, il faut reconnaître que, paradoxalement, tout en niant le mode symbolique, ces œuvres, en provoquant le spectateur, invitent à une démarche herméneutique. Devant des tableaux portant des titres tels «La promesse » ou «Le viol», on ne peut faire autrement que de chercher le rapport entre ces concepts et la représentation qui en est donnée. Mais, contrairement à l'ancien paradigme allégorique, aucune signification précise et certaine n'émane de ces images, créées pour susciter l'interrogation du spectateur plutôt que pour lui apporter des réponses. Comme le note Gombrich, ces images sont «mémorables précisément parce qu'elles sont inexplicables » (1995: 591).

La position de Magritte est caractéristique du nouveau rapport aux images qui s'établit au XXe siècle. Dans le domaine du langage, une rupture radicale s'était produite quelques dizaines d'années plus tôt, avec Mallarmé et Rimbaud, fracture que l'on a décrite comme un refus de subordonner le langage à l'exigence de la référence : « cette rupture de l'alliance entre mot et monde qui constitue une des très rares révolutions authentiques de l'esprit dans l'histoire de l'Occident et qui définit la modernité elle-même » (Steiner, 1989: 121). De toute évidence, la 
peinture effectue le même mouvement de libération. Celui-ci avait certes déjà commencé au début du XXe siècle avec l'art abstrait représenté par Kandinsky, Mondrian et Malevitch, qui éliminent tout rapport à un référent identifiable. Magritte garde un référent dans ses tableaux, mais il le subvertit par des impossibilités logiques ou visuelles. Désormais libérée, l'image peut exploser dans des illusions visuelles à la façon d'Escher ou donner libre cours à l'expression de l'absurde comme dans les dessins de Saul Steinberg (1952) ou les objets introuvables de Jacques Carelman (1980). L'image n'est plus subordonnée à la logosphère ni à l'empire du sens en général. Elle s'est émancipée de sa sujétion traditionnelle à un texte qu'elle avait pour fonction d'illustrer de façon plus ou moins séduisante et qui en fixait d'autorité le sens ultime.

Tous les peintres n'ont certes pas la même suspicion que Magritte à l'égard des symboles. Salvador Dali, notamment, en fait un usage abondant. Toutefois, au lieu de recourir à des symboles codifiés et dont la signification serait communément partagée, il procède de façon subjective, en se fiant aux résonances symboliques personnelles qu'un objet ou un animal peuvent éveiller en lui. Ainsi, dans le tableau de 1931 intitulé Six apparitions de Lénine sur un piano, les fourmis seraient censées suggérer la putréfaction, tandis que le piano symboliserait la mort (Fride, 1999: 144). On est loin des valeurs stéréotypées attachées à la fourmi. Autant dire que toute lecture allégorique est d'emblée impossible, le spectateur non prévenu étant livré sans clé aucune à l'effet d'étrangeté qui se dégage du tableau.

\section{Les symboles dans la vidéosphère}

La transformation du rapport à l'image s'accentue au fur et à mesure que s'affirme l'emprise de la vidéosphère. Désormais, l'image de référence n'est plus créée par des artistes pour des mécènes ou de riches collectionneurs, mais par des graphistes, des photographes, des infographes et des artistes de tout genre pour les productions de masse : affiches, magazines, revues spécialisées, sites Web, campagnes électorales, t-shirts, etc. Le message véhiculé par ces images doit en principe arrêter le regard en jouant sur la séduction, tout en étant interprétable et aussi mémorable que possible.

L'allégorie connaîtra dès lors une renaissance grâce à la photographie et au photomontage. Il ne s'agit plus, toutefois, des personnages abstraits et conventionnels chers à l'allégorie classique.

Un modèle d'allégorie courante est la personnification de type sociologique, où divers attributs permettent de rattacher par métonymie un personnage à une catégorie socioprofessionnelle, nationale ou religieuse. Le béret et la baguette de pain suffiront ainsi à évoquer le Français moyen; le cigare et le tableau de perfor- 
mances statistiques évoqueront le personnage du businessman; le stéthoscope et la blouse blanche désigneront le médecin; le drapeau identifiera une nationalité, etc.

Ce procédé fonctionne aussi avec la photographie, même si celle-ci tend à être dominée par la description, vu que ses sujets et personnages sont pris dans la réalité. Pour réussir à transcender son référent immédiat, la photographie mettra en évidence des attributs significatifs, en exagérant leur importance, que ce soit par le cadrage ${ }^{7}$, un jeu de lumière ou un effet de surcharge. Ce dernier procédé est superbement exploité dans Ms Cling-Free (1982), de Judy Dater ${ }^{8}$ (ci-contre). La photo met en scène une femme qui s'apprête à faire le grand ménage, ainsi que l'indiquent divers attributs visant à saturer le domaine évoqué : balai, plumeau et fer à repasser dans la main gauche, boîte de poudre à lessive, ramasse-poussière et paquet de "cling-free» dans la main droite. La femme est accoutrée comme une soubrette et dotée d'une casquette à visière évoquant l'uniforme des femmes

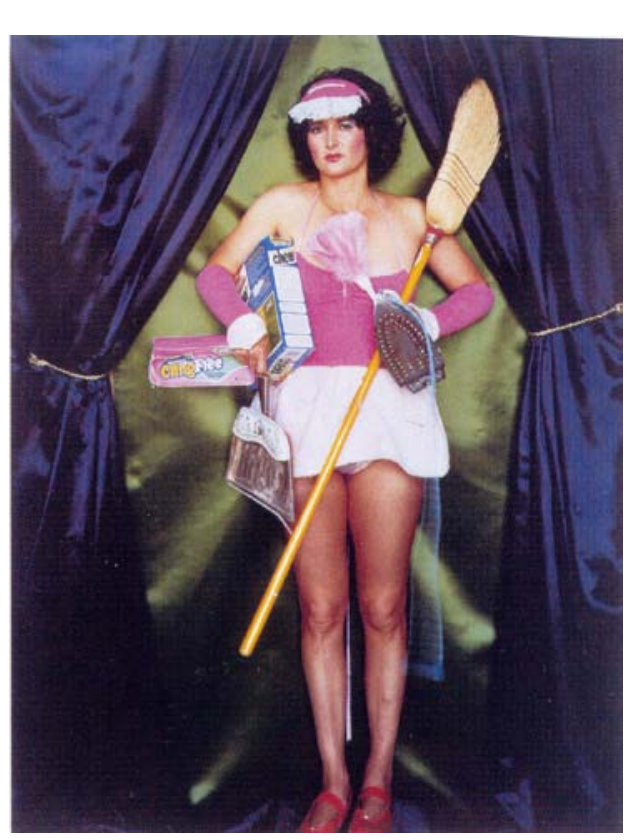
de ménage de grandes chaînes d'hôtel américaines. Pour peu que le lecteur puisse la situer dans le contexte de la fin des années 70 aux États-unis, cette photo sera immédiatement perçue comme véhiculant un message, en rapport avec le mouvement féministe qui secouait alors les stéréotypes sexistes et s'en prenait aux rôles traditionnels dévolus à la femme. L'image, dont la surcharge thématique atteste l'ironie, ne se contente pas de proposer une incarnation de la ménagère, mais prend vis-à-vis de celle-ci une position qui ne saurait échapper au lecteur le moins subtil : c'est une image de combat, une allégorie des oppressions de la vie domestique.

Plutôt que de personnifier une abstraction intemporelle, l'image symbolique contemporaine vise le plus souvent à désigner un sujet, tel le dossier thématique d'un magazine, ou à faire la publicité d'un produit. L'allégorie moderne se redéfinira ainsi comme un message visuel visant à signifier une idée abstraite ou

${ }^{7}$ Voir par exemple, Russell Lee, Les mains d'une fermière de l'Iowa, 1936.

${ }^{8}$ Cette photo a été réalisée en couleur sur Cibachrome. On en trouvera une bonne reproduction dans The Art Book, Phaidon Press, 2000, p. 108. 
un état de choses, au moyen de référents présents dans l'expérience commune du plus grand nombre.

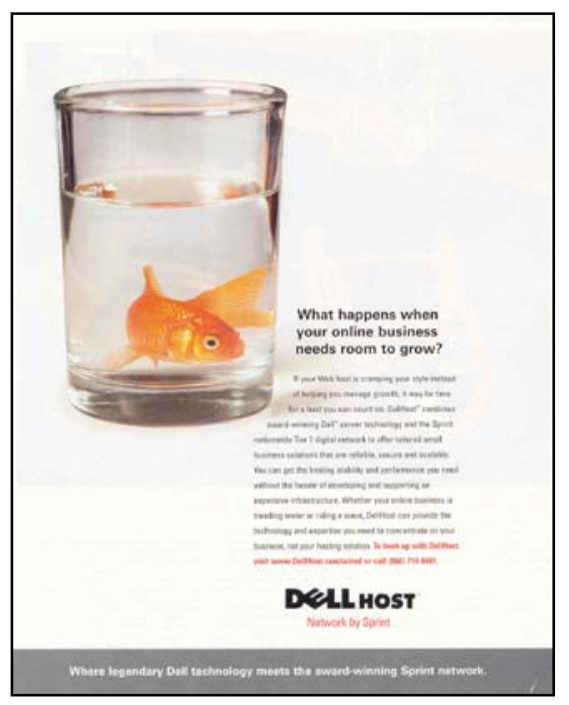

Dans une annonce publiée dans Wired (février 2004), un fournisseur de services Internet va ainsi avoir recours à un poisson rouge dans un verre pour illustrer le fait qu'il est important pour une entreprise d'avoir un espace qui lui permette de grandir. Cette allégorie limpide, qui ne nécessite pas de la part du lecteur la connaissance d'un code, fait simplement appel au sens commun. En jouant sur un rapport de proportionnalité entre la taille du poisson et celle du verre, l'image permet au lecteur d'intérioriser immédiatement le thème illustré et de se projeter en imagination dans la situation du poisson. Point n'est besoin de passer par le langage pour en décoder la signification, même si le proverbe « petit poisson deviendra grand » peut toujours refaire surface dans le savoir culturel du lecteur. Pour la personne intéressée, cette image est en outre explicitée par le texte, très détaillé, et dont la masse typographique équilibre la masse iconique. Mais ce texte est en tout petit caractère: placée en position dominante, c'est l'image qui doit d'abord retenir l'attention du lecteur et faire passer le message.

Pour illustrer un dossier sur le fossé entre l'Afrique et le monde développé, The Economist (13 mars 2004) propose en couverture un dessin où un enfant noir provenant du désert s'approche d'une cité moderne (Figure 9). Devant des gratte-ciels symbolisant la puissance de l'argent, il tient dans une main un bol, évoquant le besoin d'aliments, et dans l'autre une feuille de papier, qui doit être une liste de revendications. Le titre à la forme interrogative («A question of justice?») laisse percer l'ambivalence de ce magazine conservateur à l'égard des rapports nord-sud et place le thème du dossier dans une lumière problématique, propre à

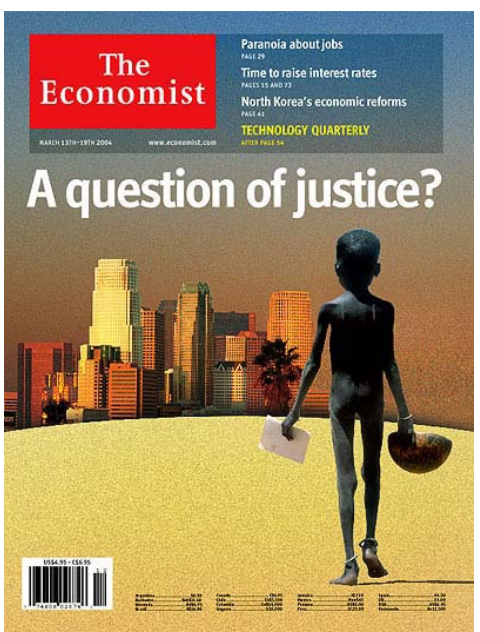
exciter la curiosité de son public habituel.

Avec ces deux derniers exemples, nous sommes loin des images allégoriques de Ripa. Chez ce dernier, les attributs n'étaient décodables qu'en vertu d'un 
lexique préétabli, formé de clichés ou de valeurs figées, du genre « vert = espérance », et accessibles aux initiés ${ }^{9}$. Dans l'allégorie moderne de grande consommation, l'image doit frapper par sa capacité de séduire et de rejoindre immédiatement l'expérience de la plupart des lecteurs, indépendamment de leur langue ou de leur bagage culturel. Elle ne joue donc plus prioritairement sur des proverbes et locutions figées, mais sur une expérience partagée du réel.

La publicité pourra toutefois recourir aussi à des symboles plus subtils et dont la signification ne saurait être déchiffrée que de façon globale et intuitive en raison de la polysémie des images retenues. Que penser, par exemple, de cette annonce pour GM publiée dans Wired (février 2004) offrant une photo de ciel d'orage, percé au loin d'une trouée lumineuse, alors qu'une route à l'avant-plan invite au voyage. En surimpression s'affiche la phrase : "The longest road in the world is the road to redemption (" Le chemin le plus long dans le monde est celui de la rédemption »). Le message linguistique signale que l'image doit être décodée

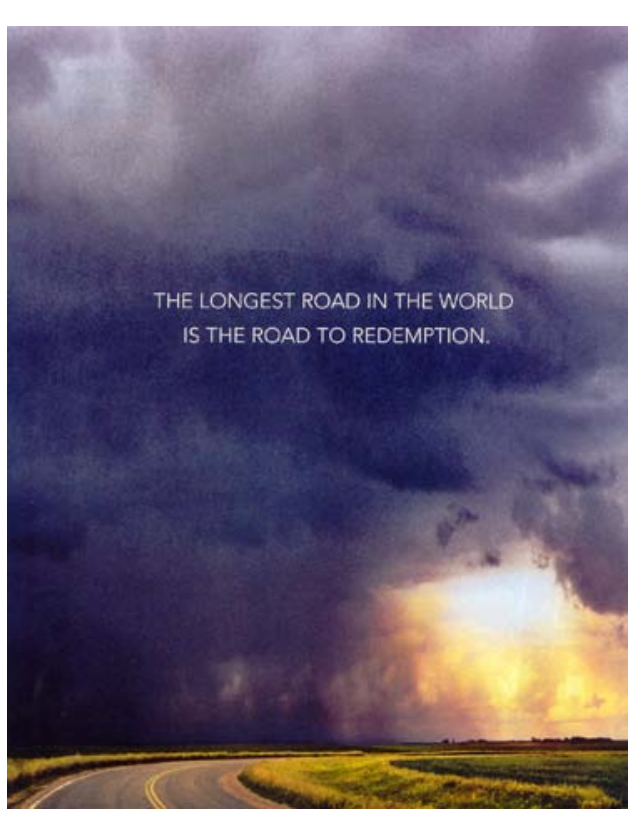
au niveau symbolique. L'ensemble s'éclaire lorsque l'on passe à la page de droite de l'encart publicitaire. Celle-ci présente trois nouveaux modèles de voitures GM et affirme que la compagnie s'est réinventée au cours des dix dernières années. Il devient alors évident que l'image en page de gauche évoque les années sombres et orageuses par lesquelles cette compagnie est passée, et qu'elle voit enfin «la lumière (au bout du tunnel)». En même temps, le terme « rédemption », que met en relief sa position en fin de phrase, évoque assurément des connotations religieuses. Celles-ci suggèrent de façon subliminale de voir l'éclaircie lumineuse comme un signe céleste préludant à une apparition divine une référence, à vrai dire, qui n'a rien de surprenant dans l'Amérique de Bush. Et le symbole est d'autant plus frappant qu'il est recontextualisé et mis au service des grands rites de consommation.

${ }^{9}$ Michel Pastoureau insiste, lui aussi, sur le fait que, à l'époque de Ripa, « savoir décoder des images c'est détenir un certain pouvoir, non seulement culturel mais aussi politique, social, religieux. » (528) 
Les jeux actuels sur le symbolisme visuel se distinguent sans doute le plus radicalement de l'allégorie classique en ceci que l'on ne crée plus de ces personnages fictifs - des hommes ou, plus souvent, des femmes - , plus grandes que nature et qui seraient censées incarner une idée, un vice ou une vertu. Les conventions qui inspiraient ces créations allégoriques dans le passé semblent même parfois ne plus être comprises. On en a eu un exemple aux Etats-Unis, en novembre 2001, lorsque le secrétaire d'État à la justice, John Ashcroft, visiblement embarrassé d'avoir été photographié auprès de la statue allégorique "l'Esprit de la Justice », l'a fait dissimuler par des tentures quelques mois plus tard ${ }^{10}$. De toute évi-

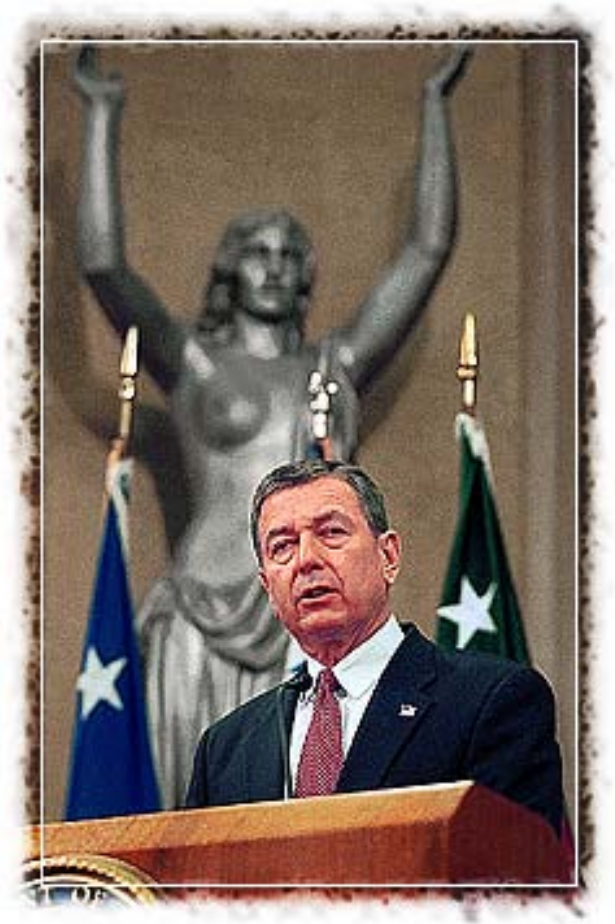
dence, ce gardien de la morale n'a pas vu dans le sein dévoilé l'idée que la Justice devrait être exempte d'artifices : il n'y a vu qu'une femme impudique.

Et peut-être cela vient-il du fait que nous n'avons plus besoin, comme jadis, de recourir à des figures emblématiques puisées dans la Bible, les vies de saints ou les récits mythiques, afin de donner une apparence humaine à des abstractions et de les rendre ainsi émouvantes ou séduisantes. L'iconographie contemporaine dispose d'un vaste répertoire de figures réelles, issues du monde du spectacle, du sport ou de la politique, et susceptibles d'évoquer, dans une communauté donnée, un trait de caractère ou un concept, pourvu que le contexte soit précisé par le texte environnant. Une dictature sanguinaire sera illustrée, au choix, par les visages de Pol Pot, Idi Amin Dada ou Saddam Hussein; le politicien tricheur, par Nixon. L'image classique de Che Guevara symbolisera la résistance à l'empire américain; celle de la fillette fuyant nue son village bombardé au napalm résumera les horreurs de la guerre du Vietnam. Même le domaine de la science a ses icônes, telle la photo d'Einstein en train de tirer la langue. Bref, notre mythologie n'est plus peuplée de dieux descendus sur terre comme dans les allégories de la Renaissance, mais d'humains chargés de leur réalité anecdotique tout en étant élevés à la dimension de types.

\footnotetext{
${ }^{10}$ Voir notamment http://www.nydailynews.com/front/story/3695p-3303c.html.
} 
En outre, nous disposons d'un vaste répertoire de figures imaginaires puisées dans les productions de la culture de masse et qui semblent vivantes dans notre esprit, parce que nous en avons suivi les aventures dans des films ou des bandes dessinées. A titre d'exemple, pour illustrer un dossier sur les nouvelles percées de la science, la revue Wired a eu recours à une figure sortie tout droit des comics ou magazines de science-fiction (août 2003). La plupart des lecteurs de cette revue auront reconnu dans ce dessin une des multiples incarnations de Superman. Les attributs ne trompent pas: cape flot-

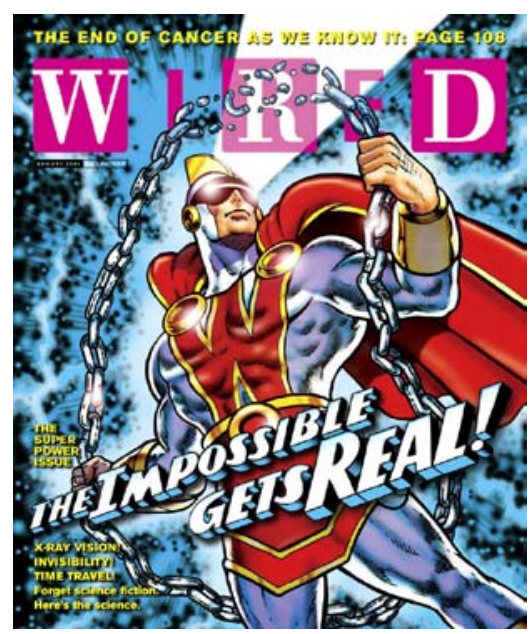
tant au vent, musculature gonflée aux stéroïdes, regard projeté vers le haut, couleurs chaudes. Combinant les figures mythiques d'Hercule et de Prométhée, ce personnage est en train de briser sans effort apparent une chaîne énorme, qui peut symboliser aussi bien les limitations de l'humanité que la chaîne de l'ADN.

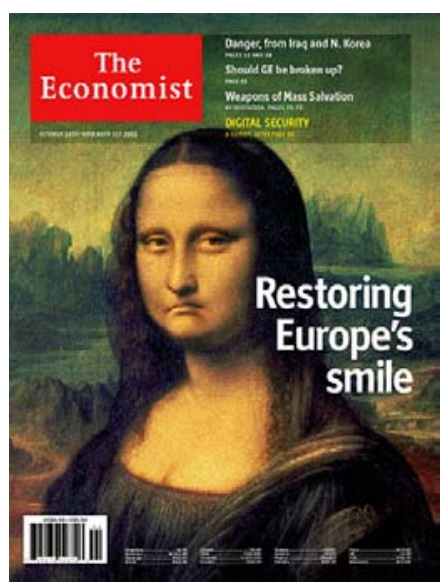

Le graphiste aura aussi recours tout simplement au patrimoine pictural, quitte à le détourner par de légères retouches. Ainsi, pour illustrer un dossier sur le climat de morosité censé régner en Europe, The Economist (26 octobre 2002) a choisi une reproduction de Mona Lisa, mais en la modifiant légèrement abstrait, grâce à une image qui, d'une part, connote la notion de haute culture, susceptible par déplacement métonymique d'être identifiée à l'Europe, et, d'autre part, joue sur la transformation du sourire légendaire en une moue non équivoque.

\section{Conclusion}

L'avènement, au XIXe siècle, de la photographie et des procédés modernes d'impression avait donné à l'image une formidable expansion et entraîné notamment la constitution du nouveau langage qu'est la bande dessinée, axée sur le narratif. La généralisation de l'ordinateur comme média universel a élargi le rôle de l'image et l'a rendue omniprésente. Non seulement, l'image peut être reproduite avec la même facilité que l'écrit et manipulée aisément, mais elle apparaît en outre nécessaire pour apprivoiser la logique froide et rébarbative des ordinateurs. Depuis le triomphe de l'interface graphique du Macintosh, elle est devenue une composante naturelle de l'affichage sur nos écrans, qui scintillent d'icônes rutilan- 
tes, de couleurs et d'images fixes ou animées. Pour circuler efficacement entre les diverses pages d'un site Web - qu'il s'agisse d'un magazine, d'une institution bancaire, d'une administration ou autre - le lecteur doit apprendre à accorder une valeur indicielle aux images rencontrées afin de s'orienter et de cliquer à bon escient : la fonction sémiotique de l'image est ainsi constamment sollicitée, bien plus que dans les médias imprimés.

Cette omniprésence des images renforce du même coup la puissance du régime symbolique, qui s'enrichit à son tour des connotations que suscitent chez le lecteur les divers contextes où une image a été utilisée. L'image est donc entrée pleinement dans un jeu d'intertextualité qui était surtout l'apanage du texte. En tant que telle, elle est en train de devenir un langage à part entière, avec tout ce que cela implique comme capacité de «dire » le réel, d'en gommer des pans entiers et, surtout, de le colorer émotivement.

Cela ne va pas sans provoquer des heurts avec le langage verbal, comme l'avait génialement pressenti René Magritte. Ces escarmouches sont maintenant devenues monnaie courante à tel point que, pour Jay David Bolter, qui a suivi dès le début les mutations du texte sous l'impact de l'ordinateur, « la relation entre le mot et l'image est en train de devenir aussi instable dans le multimédia que dans la presse populaire, et cette instabilité semble se répandre » (1996:262). Le logos ne jouissant plus de la situation de monopole qu'il occupait depuis toujours, notre civilisation doit apprendre à négocier le territoire immense du sens au moyen de la sémiotique fluide, polymorphe et séduisante des images. Confrontée au besoin de communiquer des messages qui soient compréhensibles indépendamment des langues et des substrats culturels, l'allégorie visuelle contemporaine pourrait bien jouer dans le contexte d'une société globalisée une fonction d'apprivoisement et de stabilisation. 


\section{Ouvrages cités}

Barthes, Roland (1964) «Rhétorique de l'image », L'Obvie et l'obtus, Paris : Seuil, Coll. « Essais », 1982, p. 25-42.

Benjamin, Walter (1985/1925), Origine du drame baroque allemand, Paris: Flammarion.

Bolter, Jay David (1996) «Ekphrasis, virtual reality, and the future of writing » dans G. Nunberg \& U. Eco, The future of the book, Berkeley, University of California Press, p. 253-272.

Butor, Michel (1969) Les mots dans la peinture, Genève : Skira.

Carelman, Jacques (1980) Catalogue d'objets introuvables, Paris : Balland.

Chevalier, Jean et Alain Gheerbrant, Dictionnaire des symboles, Paris, Robert Laffont et Jupiter, Coll. « Bouquins », 1982.

Debray, Régis (1992) Vie et mort de l'image, Paris : Gallimard, coll. « Folio ».

Eco, Umberto (1988) Sémiotique et philosophie du langage, Paris : PUF.

Everaert-Desmedt, Nicole (1990) Le processus interprétatif : introduction à la sémiotique de Ch. S. Peirce, Liège, Mardaga.

- - (1994) «La pensée de la ressemblance : l'œuvre de Magritte à la lumière de Peirce », Travaux du Centre des Recherches sémiologiques, 62, 85-151.

Foucault, Michel (1973) Ceci n'est pas une pipe, Montpellier : Fata Morgana.

Freud, Sigmund (1967/1900) L'interprétation des rêves. Paris : PUF.

Fride, Patricia et Isabelle Marcadé (1999) Les mouvements dans la peinture, Paris : Larousse.

Gombrich, E. H. (1995) The story of art, Londres: Phaidon.

Laneyrie-Dagen, Nadeije (2002) Lire la peinture, Paris : Larousse.

Lessing, Gotthold Ephraïm (1887) Laocoön : an essay on the limits of painting and poetry. Boston: Roberts Brothers.

Magritte, René (1994) Les mots et les images. Choix d'écrits, Bruxelles : Labor.

Pastoureau, Michel (1983) «L'illustration du livre : comprendre ou rêver? », dans Histoire de l'édition française, tome I, sous la direction de Henri-Jean MARTIN et Roger CHARTIER (dir.), p. 517-529.

Ripa, Cesare (1644/1976) Iconologie, traduit par Jean Baudoin, New York et Londres : Garland Publishing. 
Ripa, Cesare, (1618/1988) Iconologia, Edizione pratica a cura di Pietro Buscaroli, 2 vol., Turin, Fogola Editore.

Steinberg, Saul (1952) The art of living, Londres: Hamish Hamilton.

Steiner, George (1989) Réelles présences, Paris : Gallimard, NRF.

Todorov, Tzvetan (1977) Théories du symbole, Paris : Seuil.

Vandendorpe, Christian (1999) «Allégorie et interprétation », Poétique, no 117, p. 75-94.

\title{
Résumé
}

L'allégorie est un des procédés majeurs dont dispose le peintre pour faire accéder le tableau au domaine du sens. La vogue et le déclin de cette figure en peinture sont parallèles au destin changeant que connut l'allégorie verbale, tombée en discrédit à l'époque romantique. Tout comme le langage s'est détaché du

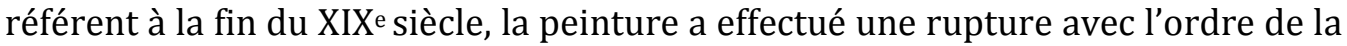
représentation quelques années plus tard. A notre époque, l'avènement de la « vidéosphère » a réintroduit dans l'image de grande consommation les exigences d'une symbolisation aussi univoque que possible. Celle-ci, toutefois, s'appuie souvent sur des procédés différents de l'allégorie classique afin de pouvoir transcender les langues et les cultures.

\begin{abstract}
Allegory is one of the most important tools at the disposal of the painter who wishes to communicate meaning through his paintings. The success and decline of allegory in painting parallels the changing fate of the verbal allegory, which fell into disregard with the advent of Romanticism. Later, just as language cut its ties to the referent at the end of the XIX'th century, painting broke with the necessity of representation in the XXth century. Today, in the age of the "videosphere", images of mass consumption are searching for ways of expressing symbols as unequivocally as possible. However, the modern allegory has had to invent new methods of expression in order to transcend languages and cultures.
\end{abstract}

